\title{
AGB Stars and Their Circumstellar Envelopes. I. the VULCAN Code
}

\author{
Sergio Cristallo ${ }^{1,2}, \mathbb{D}^{\mathbb{D}}$, Luciano Piersanti ${ }^{1,2} \mathbb{D}$, David Gobrecht ${ }^{3} \mathbb{D}$, Lucio Crivellari ${ }^{4,5}$ and Ambra Nanni ${ }^{6} \mathbb{D}$ \\ 1 INAF, Osservatorio Astronomico d'Abruzzo, Via Mentore Maggini Snc, 64100 Teramo, Italy; \\ luciano.piersanti@inaf.it \\ 2 INFN, Sezione di Perugia, Via A. Pascoli Snc, 06123 Perugia, Italy \\ 3 Institute of Astronomy, KU Leuven, Celestijnenlaan 200D, 3001 Leuven, Belgium; \\ david.gobrecht@kuleuven.be \\ 4 Instituto de Astrofisica de Canarias C/Vía Láctea, s/n E, 38205 La Laguna, Tenerife, Spain; luc_ext@iac.es \\ 5 INAF, Osservatorio di Trieste, Via Giambattista Tiepolo, 11, 34131 Trieste, Italy \\ 6 National Centre for Nuclear Research, ul. Pasteura 7, 02-093 Warszawa, Poland; ambra.nanni@ncbj.gov.pl \\ * Correspondence: sergio.cristallo@inaf.it
}

Citation: Cristallo, S.; Piersanti, L; Gobrecht, D.; Crivellari, L.; Nanni, A. AGB Stars and Their Circumstellar Envelopes. I. The VULCAN Code. Universe 2021, 7, 80. https://doi.org/ 10.3390/universe7040080

Academic Editor: Ana M. Heras

Received: 26 February 2021

Accepted: 24 March 2021

Published: 27 March 2021

Publisher's Note: MDPI stays neutral with regard to jurisdictional claims in published maps and institutional affiliations.

Copyright: (c) 2021 by the authors. Licensee MDPI, Basel, Switzerland. This article is an open access article distributed under the terms and conditions of the Creative Commons Attribution (CC BY) license (https:// creativecommons.org/licenses/by/ $4.0 /)$.

\begin{abstract}
The interplay between AGB interiors and their outermost layers, where molecules and dust form, is a problem of high complexity. As a consequence, physical processes like mass loss, which depend on the chemistry of the circumstellar envelope, are often oversimplified. The best candidates to drive mass-loss in AGB stars are dust grains, which trap the outgoing radiation and drag the surrounding gas. Grains build up, however, is far from being completely understood. Our aim is to model both the physics and the chemistry of the cool expanding layers around AGB stars in order to characterize the on-going chemistry, from atoms to dust grains. This has been our rationale to develop ab initio VULCAN, a FORTRAN hydro code able to follow the propagation of shocks in the circumstellar envelopes of AGB stars. The version presented in this paper adopts a perfect gas law and a very simplified treatment of the radiative transfer effects and dust nucleation. In this paper, we present preliminary results obtained with our code.
\end{abstract}

Keywords: AGB star; nucleosynthesis-star: abundances; reaction rate; isotopic abundance

\section{Introduction}

Asymptotic Giant Branch (AGB) stars (for reviews see [1-3]) are unparalleled laboratories for investigating the predictions of stellar evolutionary models. The structure of an AGB star consists of a partially degenerate carbon-oxygen core, a helium- and a hydrogen-burning shell separated by a thin layer (the so-called He-intershell) and a cool extended convective envelope. The latter is enriched with the products of internal nucleosynthesis (carbon, fluorine, sodium, chlorine, heavy elements), transported to the surface of the star by so called third dredge-up (TDU) episodes. Together with type Ia and type II Supernovae, chemically enriched AGB winds are therefore among the major polluters of the Inter-Stellar Medium. The amount of carbon in the envelope determines the AGB regime: $\mathrm{O}$-rich for $\mathrm{C} / \mathrm{O}<1$ and $\mathrm{C}$-rich for $\mathrm{C} / \mathrm{O}>1$. A carbon dominated chemistry has deep consequences on the physical evolution of the star, because it increases the opacity of the outermost layers, which brings about a larger expansion of the latter (see, e.g., [4]). As soon as the temperature decreases below $5000 \mathrm{~K}$, neutral atoms (re-)combine to form $\mathrm{O}$ - or C-bearing molecules of growing complexity. When the temperature drops below $1500 \mathrm{~K}$, different kinds of dust condense. This value may appear quite large. For instance, it has been suggested that silicates condensate at lower temperatures (1100-1400 K; see [5]). However, it is most likely that the dust formation in oxygen-rich AGB stars starts with condensates that are more refractory than silicates (see e.g., [6,7]). On the other hand, other authors still assume $1500 \mathrm{~K}$ as the sublimation temperature of amorphous carbon [8]. 
In summary, we are aware that the bulk of the dust presumably condenses at lower temperatures than $1500 \mathrm{~K}$. In order to reconcile with Bowen [9] we use the same dust condensation temperature of $1500 \mathrm{~K}$, but we also tested a lower dust condensation temperature of $1100 \mathrm{~K}$ (see Section 3). In the future we aim to implement a more sophisticated treatment of dust nucleation and growth. As a rule of thumb, in O-rich stars the bulk of dust production directly scales with the initial metallicity. On the other hand, in C-stars it depends on the carbon excess (i.e., on available carbon nuclei not locked into $\mathrm{CO}$ molecules), which derives from the dredge-up of fresh carbon by TDU episodes [4,10].

As a matter of fact, the process that determines the fate of low and intermediate mass stars is mass loss from their external layers, rather than nuclear burning in their interiors. The majority of material is lost during the two red giant phases: the Red Giant Branch (RGB), and the AGB. During the RGB phase, mass loss is thought to be driven by surface magnetic fields [11], while during the AGB phase the interplay between pulsation shocks and dust may be the dominant driver of the wind that expels the outermost layers (see, e.g., [12,13]). AGB mass loss rates vary from $10^{-8}$ to $10^{-4} \mathrm{M}_{\odot} / \mathrm{yr}$. Mass loss influences both physical (final core mass, AGB lifetime) and chemical properties (final isotopic composition, total yields) of an AGB star. We use the FUNS code [2] to compute our stellar evolutionary models, whose chemical and physical properties are available online on the FRUITY database (fruity.oa-teramo.inaf.it accessed on 25 March 2011) [14-17]. In FUNS, theoretical and empirical relations are employed in order to derive a prescription on mass loss by correlating the stellar pulsation period to the surface luminosity and temperature variations. It is well known that during the AGB phase, external stellar layers may become unstable against large amplitude pulsations. Major giant branch pulsator classes are semi-regular and Mira variables. In the present work, we will concentrate on the latter class.

Mira variables show large amplitude radial variations. Moreover their spectra are characterized by complex velocity structures, most likely due to the interplay between hot shocks moving outwards and cooling material falling inwards. The nature of the mechanism that triggers the pulsations is still a matter of debate, although convection is convincingly proposed as driving force [18]. Whatever the driving mechanism of the pulsations is, they induce compression of the gas and trigger the formation of recurrent shock waves, which propagate through the atmosphere and the outer layers. Shock waves lift and compress material, resulting in a higher density at lower temperature (due to the increased distance to the stellar surface). Shock-induced jumps in the temperature are leveled out efficiently by radiative cooling. The condition required for effective grain growth is an enhancement of density at cool temperatures. Under such conditions grains can absorb or scatter stellar photons $[12,13,19]$. Moreover, by colliding with atoms and molecules in the gas phase, grains transfer their momentum to the latter, driving the wind. Different types of grains, in both the C-rich and the O-rich regimes, determine the optical and dynamical properties of the stellar layers in which they form [20-24]. Despite the fact that dominating species have been identified, their nucleation path and growth are far from being understood.

The global picture resulting stresses clearly that many mutually interacting processes are at play in AGB stars: TDU mixing episodes, convection (eventually coupled to nuclear burning, as it occurs during Hot Bottom Burning), pulsations, mass-loss, dust nucleation. Since these processes are characterized by very different environmental physical conditions, two alternative approaches have been envisaged: the first centered on the physics of stellar interiors and atmospheres, the second on the physics of circumstellar envelopes (hereafter CSEs). The aim of our project is to reproduce on theoretical grounds the nucleation path of chemical species, starting from single nuclei up to the formation of dust grains. In the past, detailed chemical-kinetic models have been developed, but either they consider constant gas conditions [25,26], or have been applied to trajectories based on simplified solutions of the hydrodynamic equations [7]. In contrast to these chemical models, hydrodynamic models of great complexity were constructed, but simplified chemical equilibrium and/or classical nucleation theory was assumed to model the molecule 
and dust synthesis $[13,24,27,28]$. However, to our knowledge, there are no self-consistent models of inner AGB winds that include both an extensive hydrodynamic model and a kinetic network describing the formation and destruction of molecules and dust.

In order to attain this goal, we develop (and we are currently implementing) the "ab initio" in-house code VULCAN. Before introducing a detailed treatment of molecules and dust formation in VULCAN, we checked its soundness by comparing our results with the work proposed by [9]. The present paper, the first of a series, is organized as follows: in Section 2 we describe the features of the code and the physics it takes into account. In Section 3 we present VULCAN preliminary results, while our conclusions are summarized in Section 4.

\section{The VULCAN Code}

VULCAN is a 1D Lagrangian hydrodynamic code, written in FORTRAN90. In this first release we adopt the same assumptions and approximations introduced by Bowen [9] in his code. More sophisticated treatments (including, e.g., the solution of the radiative transfer equations and the formation of dust grains) are available in the literature ([29,30] and successive papers). Nevertheless we considered that Bowen's approach is the best one for writing ab initio our code.

Within VULCAN we assume spherical symmetry and treat shocks with the customarily used approach of pseudo-viscosity. The contributions of rotation and/or magnetic fields are ignored. The hydrodynamic equations, i.e., mass conservation (Equation (1)), momentum conservation (Equation (2)) and energy conservation (Equation (3)), are solved explicitly in a Lagrangian scheme with a standard finite-difference method:

$$
\begin{gathered}
m(r, t)=m\left(r_{0}, 0\right) \\
\frac{D u}{D t}=-4 \pi r^{2} \frac{\partial P}{\partial m}-\frac{u_{q}}{\rho}-G \frac{m}{r^{2}}+\left(\frac{k_{D} L}{4 \pi r^{2} c}\right) \\
\frac{D E}{D t}=-P \frac{D V}{D t}+\epsilon_{q}+\Lambda_{e H} / \rho+Q
\end{gathered}
$$

Symbols represent:

$t=$ time;

$r=$ distance from the center;

$m=$ mass within radius $\mathrm{r}$;

$u=$ fluid velocity;

$P=$ pressure;

$u_{q}=$ specific energy due to artificial viscosity;

$\rho=$ density;

$G$ = gravitational constant;

$k_{D}=$ mean dust cross section against radiation;

$L=$ luminosity of the star;

$c=$ speed of light;

$E=$ internal specific energy;

$V=$ volume;

$\epsilon_{q}=$ energy dissipation due to artificial viscosity $\left(\mathrm{g}^{-1} \mathrm{~s}^{-1}\right)$;

$\Lambda_{e H}=$ energy contribution due to the first excitation of hydrogen;

$Q=$ thermal relaxation to the equilibrium temperature by radiative transfer.

For the artificial viscosity we adopt the formulation of [31], who used the prescriptions in [32]. Thus in spherical coordinates artificial viscosity is given by

$$
q=3 \lambda^{2} \rho \frac{\partial\left(r^{3} u\right)}{\partial r^{3}}\left(\frac{\partial u}{\partial r}-\frac{\partial\left(r^{3} u\right)}{\partial r^{3}}\right)
$$


if $\frac{\partial\left(r^{3} u\right)}{\partial r^{3}}>0$ and 0 otherwise. This assures that pseudo-viscosity pressure is added in compressing zones only. In Equation (4), $\lambda$ represents the width of the shock front and it is usually assumed proportional to the number of meshes within the shock front: $\lambda=\sqrt{c_{q}} \Delta r$, where $\Delta r$ is the radius extension of the mesh. In our computation we set $c_{q}=2$. Further quantities appearing in Equations (2) and 3 are defined as:

$$
\begin{gathered}
u_{q}=-\frac{3}{r} \frac{\partial\left(r^{3} q\right)}{\partial r^{3}} \\
\epsilon_{q}=-\frac{1}{\rho} q\left(\frac{\partial u}{\partial r}-\frac{u}{r}\right)
\end{gathered}
$$

The equation of state assumed is that for an ideal mono-atomic gas with constant $\mu=1.26$ and $\gamma=5 / 3$ (we maintain the same assumptions as in [9] for comparison's sake).

The quantity $\Lambda_{e H}$ represents the energy contribution due to the first excitation of hydrogen, whose rate has been taken from [33], that is

$$
\Lambda_{e H}=7.3 \cdot 10^{-19} n_{e} n(H I) e^{-118400 / T}
$$

where $n(H I)$ is the number of neutral $\mathrm{H}$ atoms.

The term $Q$ in Equation (3) represents the thermal relaxation to the equilibrium temperature by radiative transfer and, following [9], it is expressed as

$$
Q=-\frac{3 R}{2 \mu}\left(T-T_{e q}\right) \frac{\rho}{C_{R}} \Delta t,
$$

where $R$ is the gas constant $\left(\mathrm{R}=8.314 \cdot 10^{7} \mathrm{erg} \mathrm{K}^{-1} \mathrm{~mol}^{-1}\right)$ and $\mu$ is the mean molecular weight. The quantity $T_{e q}$ is the radiative equilibrium temperature and it is calculated adopting the Eddington approximation for a grey atmosphere [34], that is

$$
T_{e q}=T_{e f f}\left(W+\frac{3}{4} \tau\right)^{\frac{1}{4}}
$$

where $T_{\text {eff }}$ is the photospheric temperature and $W$ is the geometrical dilution factor, defined as $W=\frac{1}{2}\left[1-\left(1-\left(R_{\text {phot }} / r\right)^{2}\right)^{\frac{1}{2}}\right]$ for radii larger than the photospheric radius $R_{\text {phot }}$. The quantity $\tau$ is the optical depth, defined as

$$
\tau=\int_{r}^{\infty}\left(R_{\text {phot }} / r\right)^{2} \kappa \rho \mathrm{d} r
$$

and $\kappa$ is the constant opacity set equal to $2 \cdot 10^{-4} \mathrm{~cm}^{2} \mathrm{~g}^{-1}$. The parameter $C_{R}=10^{-5} \mathrm{~g} \mathrm{~s} \mathrm{~cm}^{-3}$ is taken from [9].

The cooling term $Q$ in Equation (3) depends on the collisional excitation of hydrogen atoms; it leads to an almost isothermal behavior in the dense inner region, to an adiabatic one in the outermost layers.

The effects of radiation pressure on dust are included by adding the further contribution between parentheses in the right side of Equation (2). Note that $20 \%$ of stellar radiation is assumed to escape from the structure in order to take into consideration the radiation losses due to Ly $\alpha$ radiation and Balmer radiation (see [9]).

Following [9] we define:

$$
k_{D}=k_{\max } \frac{1}{1+\exp \left[\left(T_{e q}-T_{\text {cond }} / \sigma\right)\right]},
$$

where $T_{\text {cond }}$ is the dust condensation temperature (assumed equal to $1500 \mathrm{~K}$ ) and $\delta$ a parameter that defines the range of condensation $(\delta=60 \mathrm{~K}) ; k_{\text {max }}$ is a parameter adjusted so that the ratio between radiation pressure and gravity be equal to 0.95 , representative of a weakly bound gravitational system (cf. [9]). The dynamical model presented here is a test 
case for future investigation. In spite of the crude approximations introduced (see e.g., [35]), it will be the basis for successive improved versions that will allow us to investigate the degree of dependence of the chemistry on a more realistic physical input. A consistent treatment of grain growth, with the calculation of the related dust opacities, will be object of future improvement, according to the latest prescriptions of [36]. In addition, we will include a kinetic nucleation description which is based on metal oxide clusters forming mineral dust grains [37].

The initial hydrostatic model is computed by mean of an iterative process with a IV-order Runge-Kutta method. Initial stellar parameter are: $\mathrm{M}=1.2 \mathrm{M}_{\odot}$ and $\mathrm{L}=5315 \mathrm{~L} \odot$ (this determines a stellar radius $\mathrm{R}_{*}=270 \mathrm{R}_{\odot}$, equivalent to $1.88 \times 10^{13} \mathrm{~cm}$ ). Moreover, at the photosphere, the temperature is fixed to $T_{\text {phot }}=3000 \mathrm{~K}$. The CSE is assumed to have an initial extension of 2 stellar radii.

The integration is performed from the outer mesh inwards, down to a radius coordinate equal to $0.92 R_{*}$. The initial temperature profile is reported in Figure 1 as a function of the initial stellar radius. The initial model consists of 82 meshes, whose mass extension decreases with increasing mass coordinate (from $\Delta M \sim 4 \times 10^{-2} \mathrm{M}_{\odot}$ down to $10^{-8} \mathrm{M}_{\odot}$ ). The mass coordinate of the most internal mesh is at $1.11 \mathrm{M}_{\odot}$, while the most external is at $1.2075 \mathrm{M}_{\odot}$. Being the stellar mass $\mathrm{M}_{*}=1.2 \mathrm{M}_{\odot}$, the mass of the CSE is $\mathrm{M}_{C S E}=7.5 \cdot 10^{-3} \mathrm{M}_{\odot}$. We verify that the initial model is stable and at rest.

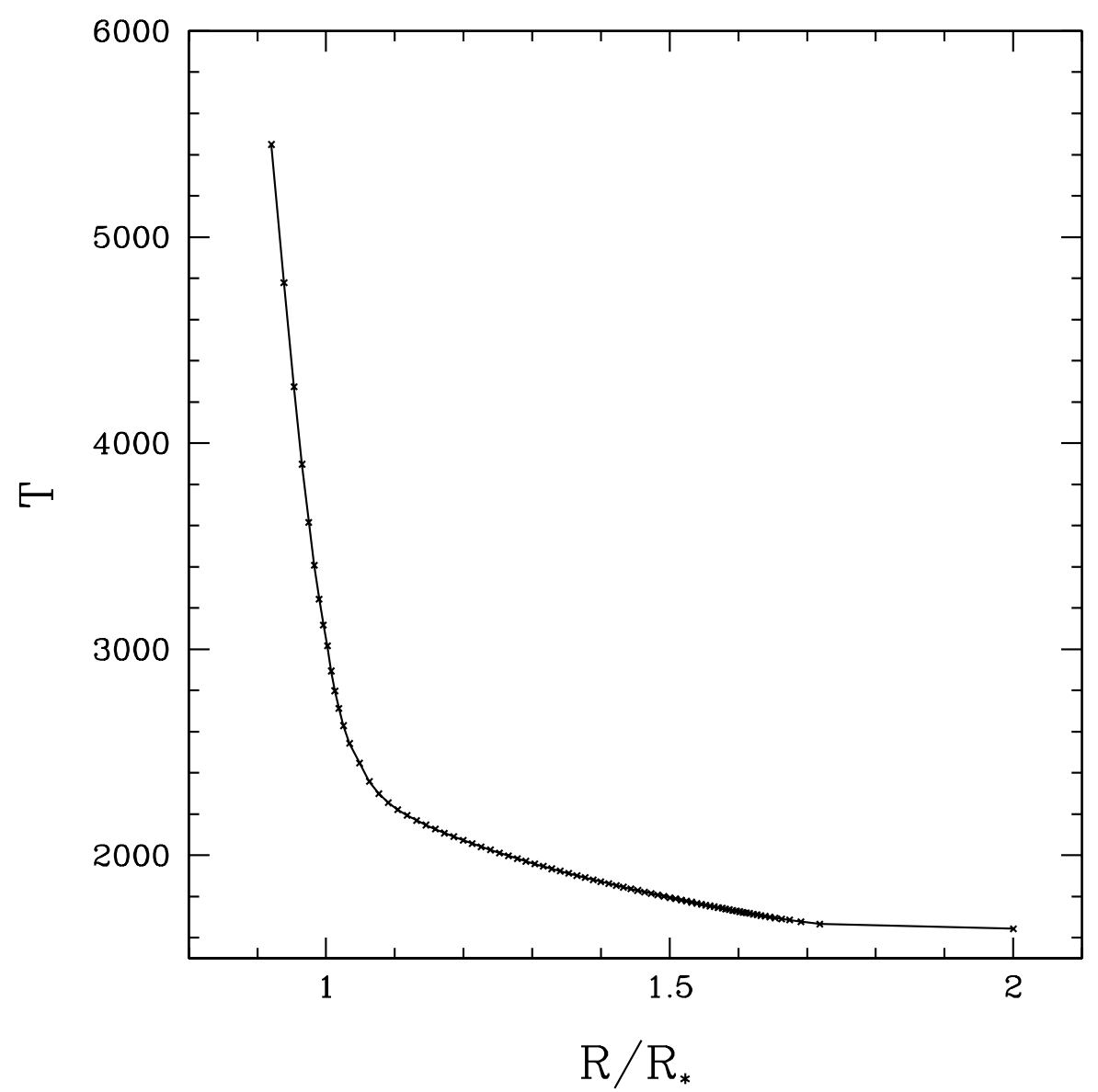

Figure 1. Temperature profile as a function of the radius of the initial hydrostatic model $\left(\mathrm{R}_{*}=270 \mathrm{R}_{\odot}\right)$.

We simulate the formation of a shock by adding a piston at the most internal mesh of our model. The velocity of the piston varies sinusoidally, as:

$$
u(0)=u_{P} \sin \left(\frac{2 \pi t}{P}\right)
$$


where $u_{P}$ is the velocity of the piston at maximum, $t$ is the time and $P$ is the oscillation period. In our Reference model we set $u_{P}=3 \mathrm{~km} / \mathrm{s}$ and $P=1 \mathrm{yr}$. The velocity of the piston is not suddenly set to its maximum amplitude, but gradually increased on a temporal interval of 20 pulsations. In such a way, strong transients are avoided (see [9]).

The propagation of shocks induces large variations of the local physical conditions in the CSE. The region above the surface is shifted repeatedly to larger radii and the radius of some meshes may result extremely stretched with respect to the initial configuration. Therefore the mesh structure of the initial model is inadequate to determine properly the local conditions of the gas at large pulsation-numbers. For this reason we implement the code with an adaptive mesh grid procedure, calibrated to achieve the best compromise between a resolution large enough and an acceptable CPU time. Our model takes into account neither the effects induced by external ultraviolet radiation nor the cooling by rotational and/or vibrational excitation of the most abundant molecules (see, e.g., [38]). Thus, we add meshes in the inner 5 stellar radii (where the contribution from any external source can be safely ignored) and we decrease the resolution in the outer region. As a consequence, the mesh resolution of the external layers results to be definitely more coarse than the inner ones. The final mesh structure consists of 450-500 grid cells (on average), most of them concentrated within the first 5 stellar radii. We ensure that the inclusion of such adaptive mesh grid does not violate the energy conservation by more than $\Delta \mathrm{E} / \mathrm{E} \sim 10^{-7}$ for the full model (300 pulsations).

\section{Results}

In Figure 2 we report the density profiles (upper panel) and the temperature profiles (lower panel) as a function of the radius for different pulsation-numbers.

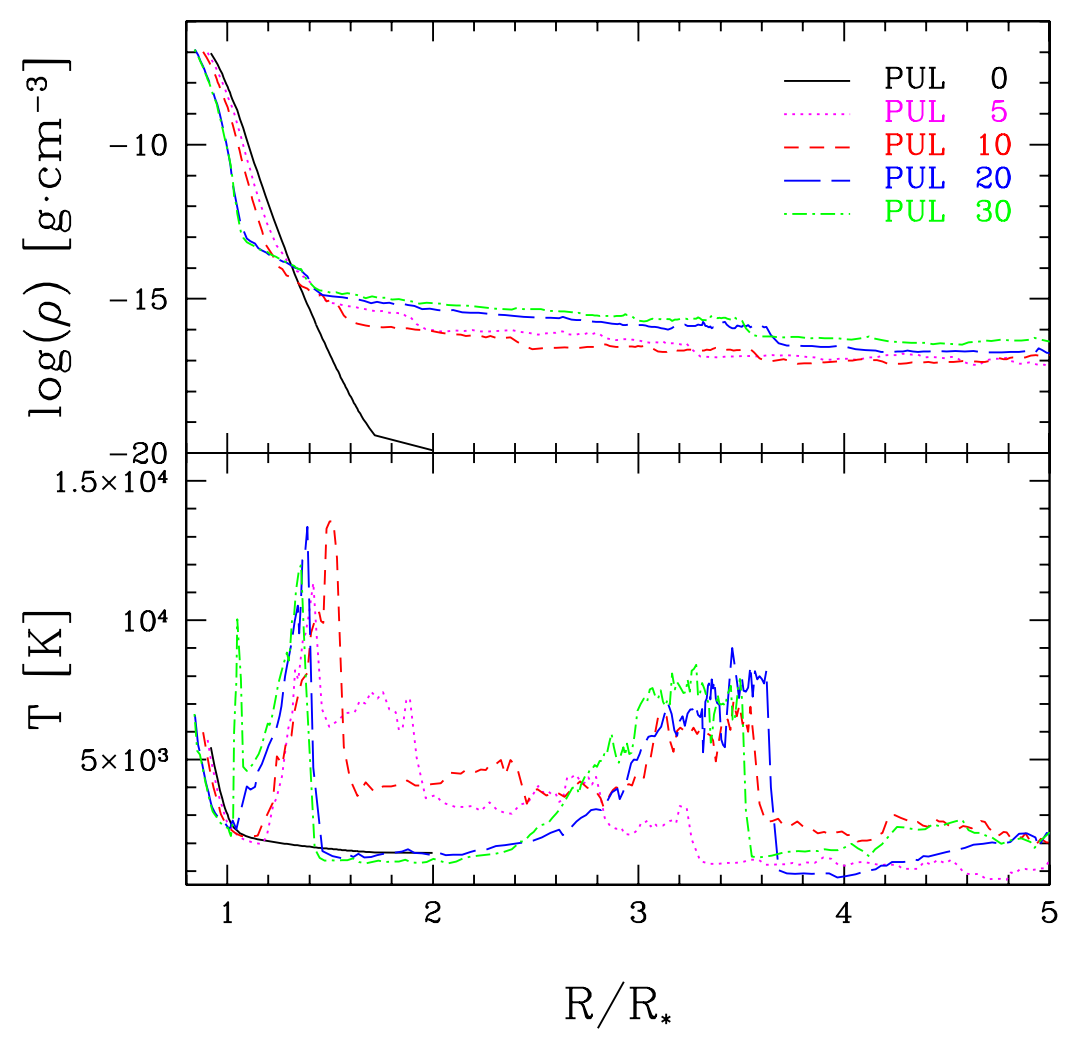

Figure 2. Density profiles (upper panel) and temperature profiles (lower panel) as a function of the radius coordinate for different pulsation-numbers. 
The effects of shocks propagation is clear. A shock front induces a compression of the gas, with a consequent increase of the local temperature and density. The density enhancement is roughly a factor 4 , thus fully consistent with the Rankine-Hugoniot equation for a mono-atomic gas (see e.g., the jump around $3.6 \mathrm{R}_{*}$ of the 20th pulsation cycle). As a net effect, matter slowly proceeds from internal to external layers, as testified by the increase of the density plateau for radii larger than $1.5 \mathrm{R}_{*}$. After the passage of the shock, the layer relaxes and, depending on its radius coordinate, returns to its position with a ballistic trajectory or it is gradually shifted toward larger radii. A closer inspection to Figure 2 reveals that, depending on the pulsation-number, the physical conditions are quite different: this demonstrates that the structure did not reach a stable periodic configuration yet, because the first 20 pulsations are damped.

In Figure 3 we report the same quantities as in Figure 2, but for advanced pulsation cycles. From this plot it clearly emerges that after 30 pulsations (i.e., after 10 normal cycles) the structure attains an asymptotic configuration, with well identified temperature peaks and extended relaxing regions.

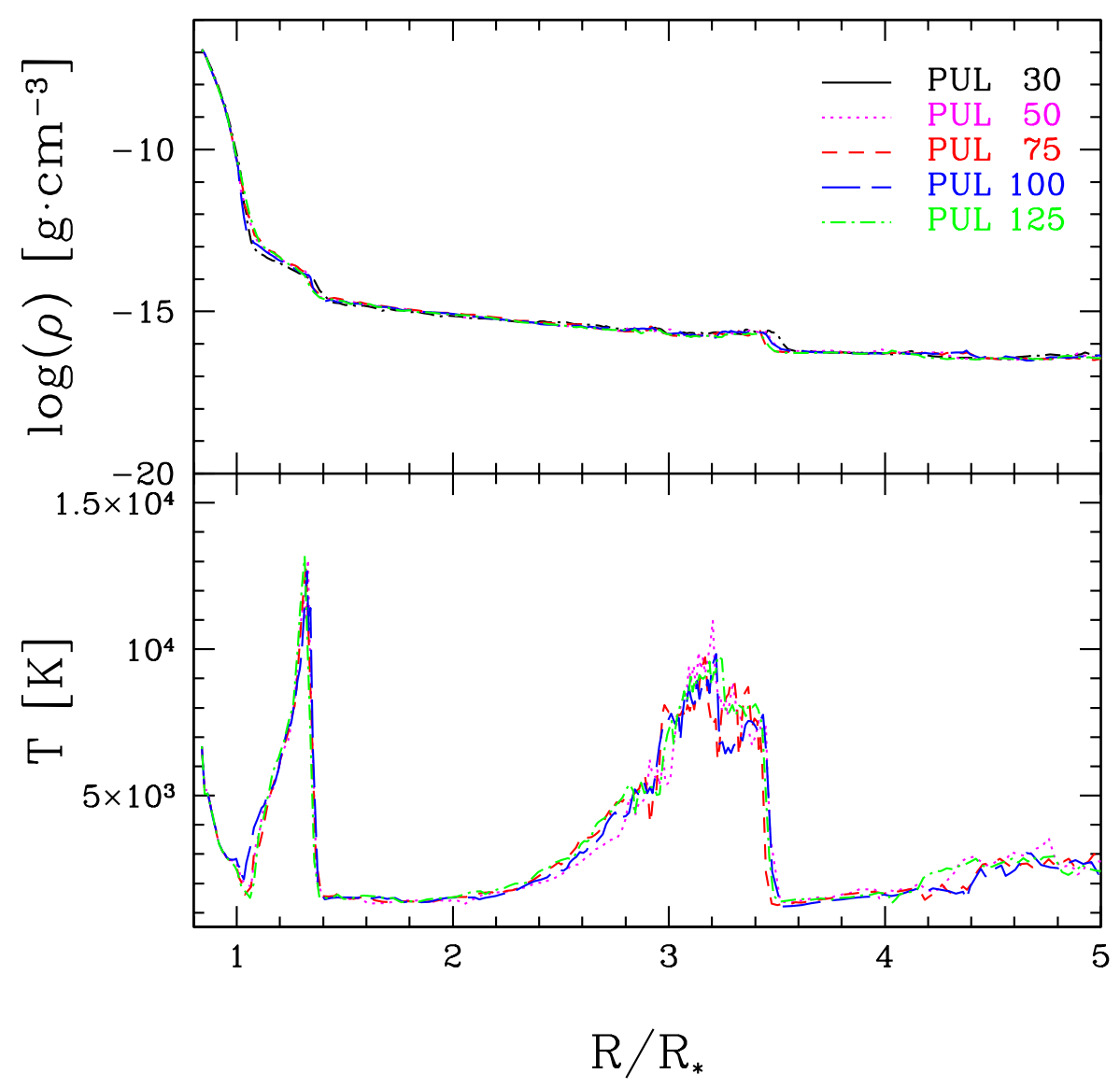

Figure 3. Same as in Figure 2, but for larger pulsation numbers.

An interesting quantity to monitor is the time evolution of the radii at given mass (Lagrangian) coordinate. In the left panel of Figure 4 we report selected Lagrangian zones, starting from the photosphere (heavy blue curve). The inner regions mildly oscillate, showing a ballistic behavior: the shock pushes those layers to larger radii, but the gravitational force pull them to their original position. The larger the initial radius, the larger the amplitude of such an oscillation. For radii larger than $2 \mathrm{R}_{*}$ (about $3.8 \cdot 10^{13} \mathrm{~cm}$ ), however, Lagrangian zones start drifting outward (due to the interaction between shocks and dust), thus driving a stellar mass-loss. 

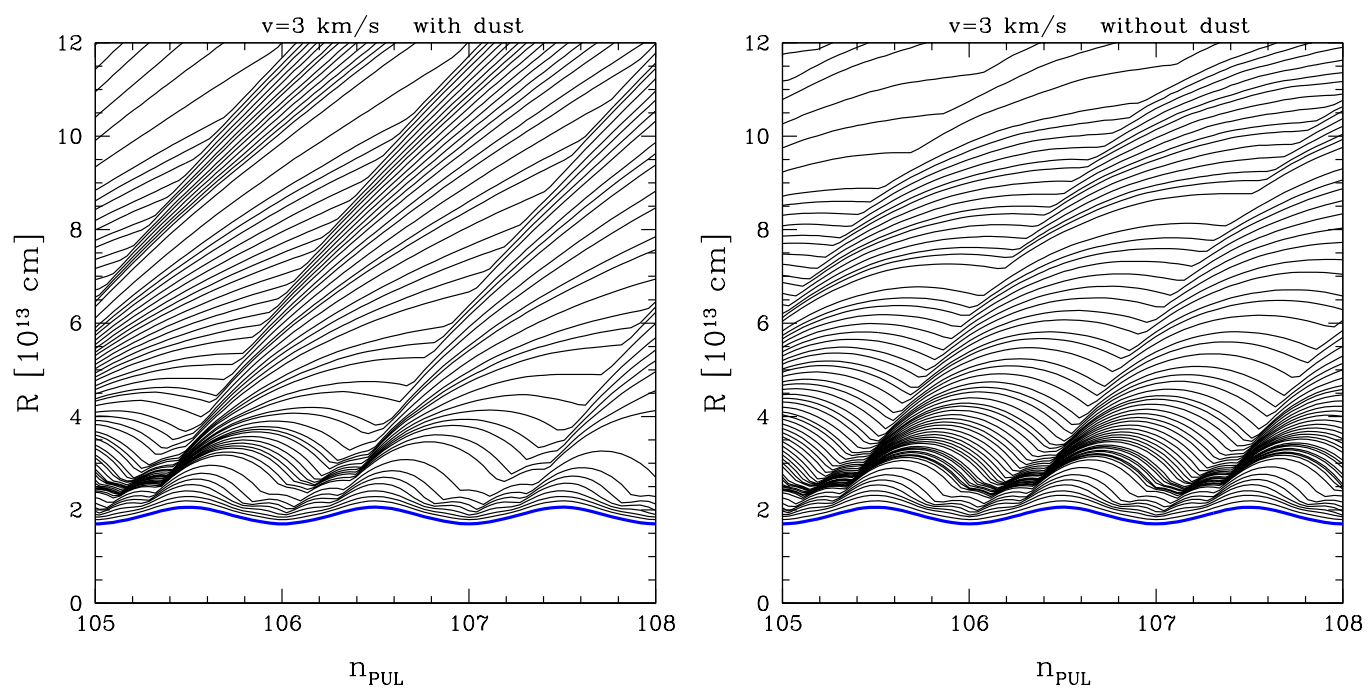

Figure 4. Temporal evolution of the radius coordinate of selected Lagrangian zones (with and without dust). Each curve follows the radial evolution of a mass shell. The innermost curve corresponds to the photosphere.

In order to evaluate the effects of dust on the time evolution of the radii, we run a model without the contribution of dust. Such a model is shown in the right panel of Figure 4. It is clear how much dust contributes to the outward drift, magnifying the deviation from a ballistic behavior. For instance, during a time interval corresponding to 3 pulsations, a zone with $R \sim 2 R_{*}$ almost increases its radius by a factor of about 2.5 for pulsation 105 in the model with dust. On the contrary, in the case without dust the radius growths of $10 \%$ only. This suggests that the winds in AGB stars could be pulsation-driven dusty winds.

Similar conclusions can be derived by analyzing the resulting mass loss rates. $\mathrm{Ob}$ served AGB mass-loss rates span a quite large range (from $10^{-8}$ to $10^{-4} \mathrm{M}_{\odot} \mathrm{yr}^{-1}$ ). Our current treatment of dust does not allow us to draw firm conclusions about mass-loss, but can point out some general features. In Figure 5 we report the mass-loss rate: we calculate for each cycle the flux of matter passing through a specific layer (we set this limit at $20 \mathrm{R}_{*}$ ).

After a transient phase (the first 50 cycles), our Reference model (labelled Reference) attains an asymptotic regime, with a mean mass-loss rate of about $6 \times 10^{-8} \mathrm{M}_{\odot} \mathrm{yr}^{-1}$. This number is lower by a factor three than the value reported by [9] for a model with the same input parameters (see the black arrow in Figure 5). This is probably due to the slightly different physics recipes introduced in the two codes (as for the pseudoviscosity). In the same plot we also present the model without dust contribution (labelled No dust). As expected, it shows a reduced mass-loss rate (a factor 8 lower with respect to the Reference case). Moreover, we also run a case with a reduced dust condensation temperature $\left(\mathrm{T}_{\text {cond }}=1100 \mathrm{~K}\right)$. This model shows an intermediate mass-loss rate between the reference model and the case without dust. This is presumably due to the fact that the choice of a lower temperature shifts the dust condensation layer to larger radii, where the density is (on average) lower and, thus, the mass-loss is less efficient. We demand to a future paper a deep analysis of this argument, also considering that we aim at substituting the current oversimplified dust treatment with a more meaningful implementation of dust nucleation in the future versions of the code. In Figure 5 we also show two additional models, representative of extreme regimes: the adiabatic case (labelled Adiabatic) and the isothermal case (labelled Isothermal). In the latter case the condition $T=T_{e q}$ holds for the whole structure. Thus no heat is transferred from shocks to the gas and the resulting mass-loss rate is very low $\left(\dot{M}<10^{-12} \mathrm{M}_{\odot} \mathrm{yr}^{-1}\right)$. In the former case the cooling term $Q$ is always zero; thus more energy is transferred to the gas, which is pushed outward more efficiently (in this case $\dot{M}>3 \times 10^{-6} \mathrm{M}_{\odot} \mathrm{yr}^{-1}$ ). 


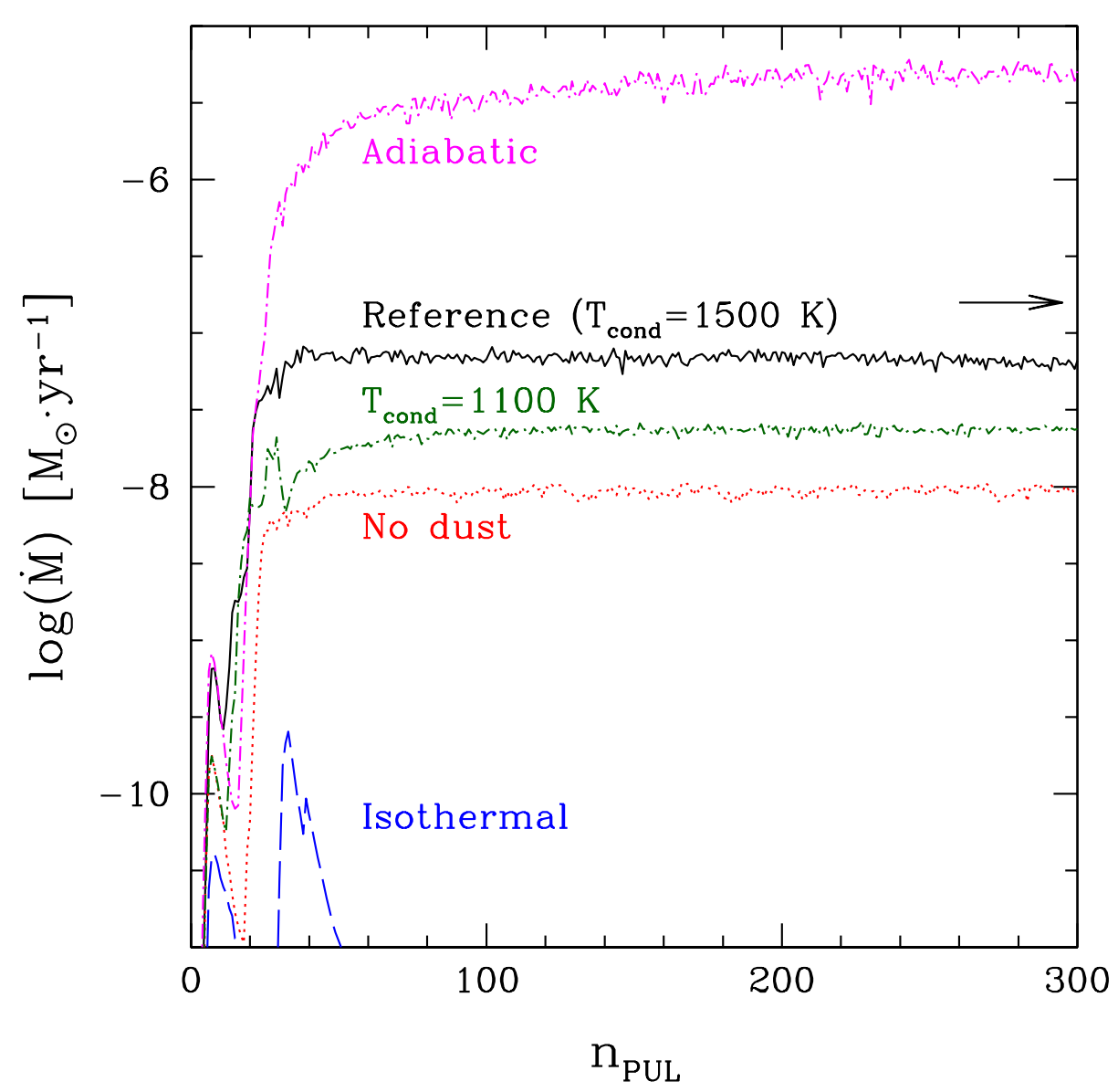

Figure 5. Theoretical mass-loss rates as a function of the pulsation number. See text for details.

In Figure 6 we present the velocity profiles as a function of the radius coordinate for the Reference model and the model without dust. For both cases we select an advanced phase, in which the mass-loss has already reached its asymptotic regime.

$\left(n_{P U L}>200\right)$. The model without dust (dotted curve) attains a terminal gas expansion velocity of about $2 \mathrm{~km} / \mathrm{s}$ (see [9] for a detailed explanation of the physics of the mass-loss in the No dust case). The presence of dust produces larger terminal velocities $(>10 \mathrm{~km} / \mathrm{s})$, due to the interaction between grains and the radiation field. Both values fairly agree with results presented in [9]. A comparison with the observations, however, is more meaningful. Information on the terminal outflow velocity of the gas can be obtained from the analysis of $\mathrm{CO}$ thermal radio emission and/or by maser emission. The velocity profile of our model with dust is consistent with the observations, which show a spread between 10 and $20 \mathrm{~km} / \mathrm{s}$ (see, e.g., [39-41]). The values obtained are also consistent with the typical expansion velocity derived for stars in our Galaxy [42-44] and in the Large Magellanic Cloud $[45,46]$. A consistent treatment of dust growth and dust-driven wind e.g., $[13,47,48]$ will be implemented in the future. 


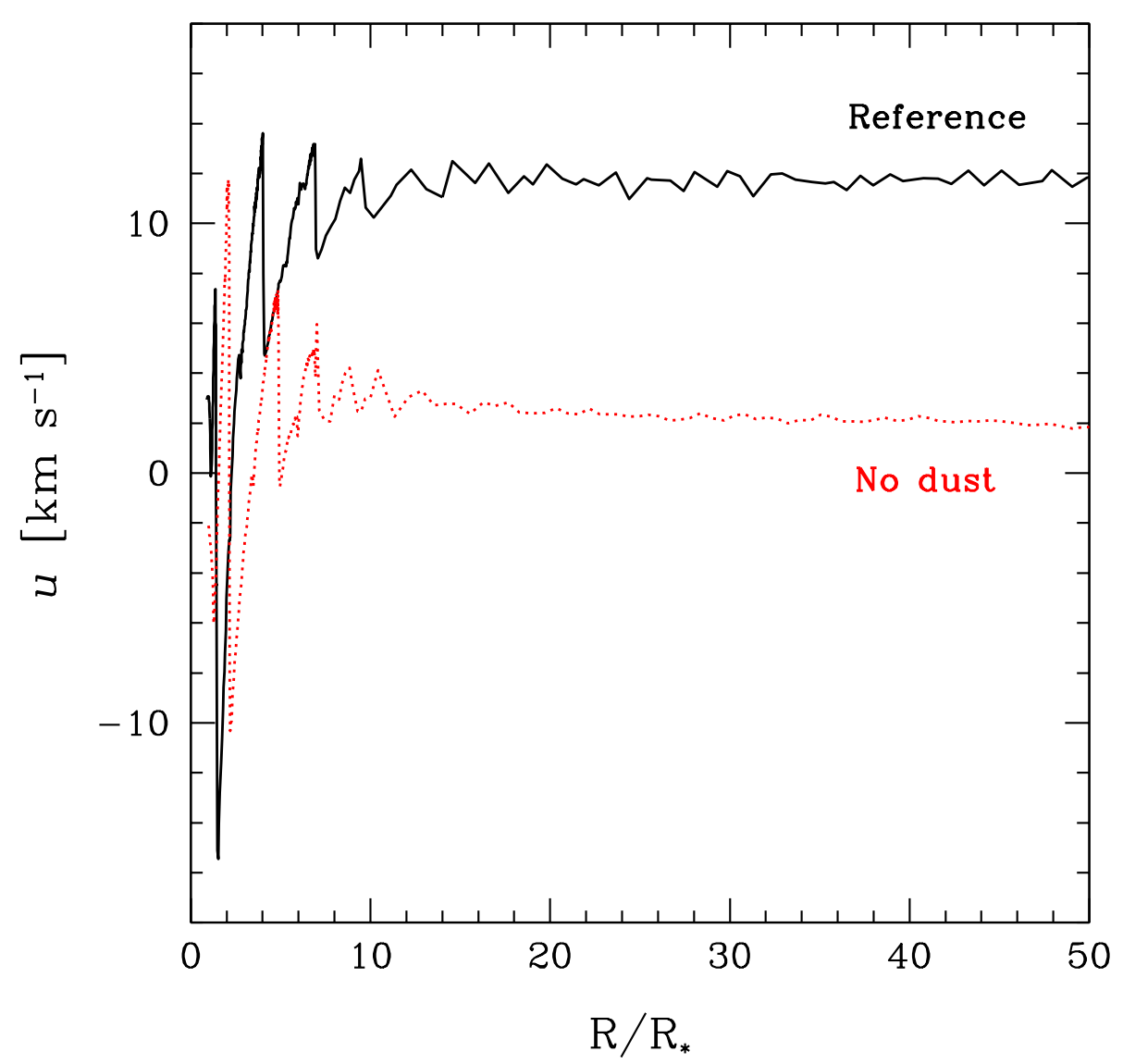

Figure 6. Velocity profiles as a function of the radius coordinate for the Reference model and for the model without dust.

\section{Conclusions}

In this paper, the first of a series, we have presented our code VULCAN, a 1D lagragian hydrodynamic code that pictures the propagation of shocks in CSEs. VULCAN is intended as a preliminary step, in view of necessary further developments that shall take into account the physical processes mentioned above. At the present stage we introduced crude simplifying hypotheses, especially in the treatment of radiative transfer. For instance, following [9], the cooling (or heating) of matter via radiative losses or the estimate of the pressure exerted by radiation on dust grains. In order to go beyond these shortcomings we are working to implement into a new version of the code the solution of the radiative transfer (RT) equation in spherical geometry by means of the Implicit Integral Method described in [49]. The ultimate goal is to compute a physical sound structure of the outer stellar layers via the self-consistent solution of the hydrodynamic and RT equations under the constraint of energy conservation [50]. We will test this new subroutine by mimicking the propagation of shocks with the injection of a prescribed amount of non-radiative energy at given depths in the outermost layers. Another substantial improvement will be to replace the equation of state (EOS) for an ideal perfect gas with constant $\mu$ and $\gamma$ with a more realistic EOS, in which the principal molecules that determine the thermodynamic properties in the range $1000<T<4000 \mathrm{~K}$ are taken into account.

Another important topic, strictly related to the RT problem, is the determination of the nucleation path of various dust species. Our aim is to couple a non-equilibrium chemistry of large gas-phase clusters to different nucleation schemes including standard dust growth theory on pre-existing seed nuclei. As a matter of fact, Classical Nucleation Theory can properly describe the growing path of dust species, but it is inappropriate to 
mimic the nucleation process and the formation of critical seed nuclei. An intermediate step, that is the synthesis of large clusters of molecules via a non-equilibrium network, is therefore required. For this reason we are actually implementing a chemical-kinetic network able to follow the synthesis of molecular species, from single atoms to complex clusters of molecules. The latter will be the seeds for the formation of dust grains that will proceed through accretion of molecules from the gas phase [5,51]. The accretion of dust grains has been shown to be critically important for stellar mass-loss [8]. Dust growth will therefore be coupled with a consistent description of dust-driven wind in the future versions of VULCAN.

Author Contributions: Conceptualization, S.C., L.P. and D.G.; methodology, S.C.; software, L.P.; validation, D.G., L.C. and A.N.; formal analysis, S.C.; investigation, S.C.; resources, S.C.; data curation, S.C., L.P. and D.G.; writing-original draft preparation, S.C.; writing-review and editing, S.C. and L.C.; visualization, S.C., D.G., L.C. and A.N.; supervision, S.C.; project administration, S.C.; funding acquisition, S.C. All authors have read and agreed to the published version of the manuscript.

Funding: DG acknowledge support from the ERC consolidator grant number 646758. AN acknowledges support from the Narodowe Centrum Nauki (UMO-2018/30/E/ST9/00082 and UMO2018/30/M/ST9/00757).

Institutional Review Board Statement: Not applicable.

Informed Consent Statement: Not applicable.

Conflicts of Interest: The authors declare no conflict of interest.

\section{References}

1. Herwig, F. Evolution of Asymptotic Giant Branch Stars. ARAEA 2005, 43, 435-479. [CrossRef]

2. Straniero, O.; Gallino, R.; Cristallo, S. s process in low-mass asymptotic giant branch stars. Nucl. Phys. A 2006, 777, 311-339. [CrossRef]

3. Karakas, A.I.; Lattanzio, J.C. The Dawes Review 2: Nucleosynthesis and Stellar Yields of Low- and Intermediate-Mass Single Stars. PASA 2014, 31, e030. [CrossRef]

4. Marigo, P. Asymptotic Giant Branch evolution at varying surface C/O ratio: Effects of changes in molecular opacities. AEA 2002, 387, 507-519. [CrossRef]

5. Nanni, A.; Bressan, A.; Marigo, P.; Girardi, L. Evolution of thermally pulsing asymptotic giant branch stars-II. Dust production at varying metallicity. MNRAS 2013, 434, 2390-2417. [CrossRef]

6. Gail, H.P.; Wetzel, S.; Pucci, A.; Tamanai, A. Seed particle formation for silicate dust condensation by SiO nucleation. AEA 2013, 555, A119. [CrossRef]

7. Gobrecht, D.; Cherchneff, I.; Sarangi, A.; Plane, J.M.C.; Bromley, S.T. Dust formation in the oxygen-rich AGB star IK Tauri. A\&A 2016, 585, A6. [CrossRef]

8. Höfner, S.; Olofsson, H. Mass loss of stars on the asymptotic giant branch. Mechanisms, models and measurements. AEA Rev. 2018, 26, 1. [CrossRef]

9. Bowen, G.H. Dynamical modeling of long-period variable star atmospheres. ApJ 1988, 329, 299-317. [CrossRef]

10. Cristallo, S.; Straniero, O.; Lederer, M.T.; Aringer, B. Molecular Opacities for Low-Mass Metal-poor AGB Stars Undergoing the Third Dredge-up. ApJ 2007, 667, 489-496. [CrossRef]

11. Dupree, A.K.; Hartmann, L.; Avrett, E.H. Chromospheres and mass loss in metal-deficient giant stars. ApJ 1984, 281, L37-L39. [CrossRef]

12. Nowotny, W.; Höfner, S.; Aringer, B. Line formation in AGB atmospheres including velocity effects. Molecular line profile variations of long period variables. $A \mathcal{E} A$ 2010, 514, A35. [CrossRef]

13. Höfner, S. Winds of M-type AGB stars driven by micron-sized grains. AEA 2008, 491, L1-L4. [CrossRef]

14. Cristallo, S.; Piersanti, L.; Straniero, O.; Gallino, R.; Domínguez, I.; Abia, C.; Di Rico, G.; Quintini, M.; Bisterzo, S. Evolution, Nucleosynthesis, and Yields of Low-mass Asymptotic Giant Branch Stars at Different Metallicities. II. The FRUITY Database. ApJS 2011, 197, 17. [CrossRef]

15. Piersanti, L.; Cristallo, S.; Straniero, O. The Effects of Rotation on s-process Nucleosynthesis in Asymptotic Giant Branch Stars. ApJ 2013, 774, 98. [CrossRef]

16. Cristallo, S.; Straniero, O.; Piersanti, L.; Gobrecht, D. Evolution, Nucleosynthesis, and Yields of AGB Stars at Different Metallicities. III. Intermediate-mass Models, Revised Low-mass Models, and the ph-FRUITY Interface. ApJS 2015, 219, 40. [CrossRef]

17. Cristallo, S.; Karinkuzhi, D.; Goswami, A.; Piersanti, L.; Gobrecht, D. Constraints of the Physics of Low-mass AGB Stars from CH and CEMP Stars. ApJ 2016, 833, 181. [CrossRef] 
18. Freytag, B.; Liljegren, S.; Höfner, S. Global 3D radiation-hydrodynamics models of AGB stars. Effects of convection and radial pulsations on atmospheric structures. AEA 2017, 600, A137. [CrossRef]

19. Bladh, S.; Höfner, S. Exploring wind-driving dust species in cool luminous giants. I. Basic criteria and dynamical models of M-type AGB stars. AEA 2012, 546, A76. [CrossRef]

20. Mattsson, L.; Wahlin, R.; Höfner, S. Dust driven mass loss from carbon stars as a function of stellar parameters . I. A grid of solar-metallicity wind models. AEA 2010, 509, A14. [CrossRef]

21. Sloan, G.C.; Matsuura, M.; Lagadec, E.; van Loon, J.T.; Kraemer, K.E.; McDonald, I.; Groenewegen, M.A.T.; Wood, P.R.; Bernard-Salas, J.; Zijlstra, A.A. Carbon-rich Dust Production in Metal-poor Galaxies in the Local Group. ApJ 2012, $752,140$. [CrossRef]

22. Eriksson, K.; Nowotny, W.; Höfner, S.; Aringer, B.; Wachter, A. Synthetic photometry for carbon-rich giants. IV. An extensive grid of dynamic atmosphere and wind models. AEA 2014, 566, A95. [CrossRef]

23. Boyer, M.L.; McQuinn, K.B.W.; Barmby, P.; Bonanos, A.Z.; Gehrz, R.D.; Gordon, K.D.; Groenewegen, M.A.T.; Lagadec, E.; Lennon, D.; Marengo, M.; et al. An Infrared Census of DUST in Nearby Galaxies with Spitzer (DUSTiNGS). II. Discovery of Metal-poor Dusty AGB Stars. ApJ 2015, 800, 51. [CrossRef]

24. Höfner, S.; Bladh, S.; Aringer, B.; Ahuja, R. Dynamic atmospheres and winds of cool luminous giants. I. $\mathrm{Al}_{2} \mathrm{O}_{3}$ and silicate dust in the close vicinity of M-type AGB stars. AEA 2016, 594, A108. [CrossRef]

25. Gobrecht, D.; Cristallo, S.; Piersanti, L.; Bromley, S.T. Nucleation of Small Silicon Carbide Dust Clusters in AGB Stars. ApJ 2017, 840, 117. [CrossRef]

26. Boulangier, J.; Gobrecht, D.; Decin, L.; de Koter, A.; Yates, J. Developing a self-consistent AGB wind model - II. Non-classical, non-equilibrium polymer nucleation in a chemical mixture. MNRAS 2019, 489, 4890-4911. [CrossRef]

27. Winters, J.M.; Le Bertre, T.; Jeong, K.S.; Helling, C.; Sedlmayr, E. A systematic investigation of the mass loss mechanism in dust forming long-period variable stars. AEA 2000, 361, 641-659.

28. Woitke, P. Too little radiation pressure on dust in the winds of oxygen-rich AGB stars. AEA 2006, 460, L9-L12. [CrossRef]

29. Dorfi, E.A.; Feuchtinger, M.U. Nonlinear stellar pulsations. I-Numerical methods, basic physics, initial models and first results. $A \mathcal{E} A$ 1991, 249, 417-427.

30. Dorfi, E.A.; Hoefner, S. Dust formation in winds of long-period variables. I - Equations, method of solution, simple examples. AEA 1991, 248, 105-114.

31. Bravo, E. Nucleosintesis y Curvas de Luz en Supernovas de Tipo Ia. Ph.D. Thesis, Universidad de Barcelona, Barcelona, Spain, 1990.

32. Tscharnuter, W.M.; Winkler, K.H.A. A method for computing selfgravitating gas flows with radiation. Comput. Phys. Commun. 1979, 18, 171-199. [CrossRef]

33. Spitzer, L. Physical Processes in the Interstellar Medium; Wiley-Interscience Publication: New York, NY, USA, 1978.

34. Chandrasekhar, $\mathrm{S}$. The radiative equilibrium of extended stellar atmospheres. MNRAS 1934, 94, 444-458. [CrossRef]

35. Woitke, P.; Goeres, A.; Sedlmayr, E. On the gas temperature in the shocked circumstellar envelopes of pulsating stars. II. Shock induced condensation around R Coronae Borealis stars. AEA 1996, 313, 217-228.

36. Nanni, A.; Groenewegen, M.A.T.; Aringer, B.; Rubele, S.; Bressan, A.; van Loon, J.T.; Goldman, S.R.; Boyer, M.L. The mass-loss, expansion velocities, and dust production rates of carbon stars in the Magellanic Clouds. MNRAS 2019, 487, 502-521. [CrossRef]

37. Gobrecht, D.; Decin, L.; Cristallo, S.; Bromley, S.T. A global optimisation study of the low-lying isomers of the alumina octomer $\left(\mathrm{Al}_{2} \mathrm{O}_{3}\right)_{8}$. Chem. Phys. Lett. 2018, 711, 138-147. [CrossRef]

38. Decin, L.; Hony, S.; de Koter, A.; Justtanont, K.; Tielens, A.G.G.M.; Waters, L.B.F.M. Probing the mass-loss history of AGB and red supergiant stars from CO rotational line profiles. I. Theoretical model-Mass-loss history unravelled in VYCMa. AEA 2006, 456, 549-563. [CrossRef]

39. Ramstedt, S.; Schöier, F.L.; Olofsson, H.; Lundgren, A.A. On the reliability of mass-loss-rate estimates for AGB stars. AEA 2008, 487, 645-657. [CrossRef]

40. Bains, I.; Cohen, R.J.; Louridas, A.; Richards, A.M.S.; Rosa-González, D.; Yates, J.A. Sub-au imaging of water vapour clouds around four asymptotic giant branch stars. MNRAS 2003, 342, 8-32. [CrossRef]

41. Decin, L.; Montargès, M.; Richards, A.M.S.; Gottlieb, C.A.; Homan, W.; McDonald, I.; El Mellah, I.; Danilovich, T.; Wallström, S.H.J.; Zijlstra, A.; et al. (Sub)stellar companions shape the winds of evolved stars. Science 2020, 369, 1497-1500. [CrossRef]

42. Lagadec, E.; Zijlstra, A.A.; Mauron, N.; Fuller, G.; Josselin, E.; Sloan, G.C.; Riggs, A.J.E. The low wind expansion velocity of metal-poor carbon stars in the Halo and the Sagittarius stream. MNRAS 2010, 403, 1331-1338. [CrossRef]

43. Ramstedt, S.; Olofsson, $\mathrm{H}$. The ${ }^{12} \mathrm{CO} /{ }^{13} \mathrm{CO}$ ratio in AGB stars of different chemical type. Connection to the ${ }^{12} \mathrm{C} /{ }^{13} \mathrm{C}$ ratio and the evolution along the AGB. AEA 2014, 566, A145. [CrossRef]

44. Danilovich, T.; Teyssier, D.; Justtanont, K.; Olofsson, H.; Cerrigone, L.; Bujarrabal, V.; Alcolea, J.; Cernicharo, J.; Castro-Carrizo, A.; García-Lario, P.; et al. New observations and models of circumstellar CO line emission of AGB stars in the Herschel SUCCESS programme. AEA 2015, 581, A60. [CrossRef]

45. Groenewegen, M.A.T.; Vlemmings, W.H.T.; Marigo, P.; Sloan, G.C.; Decin, L.; Feast, M.W.; Goldman, S.R.; Justtanont, K.; Kerschbaum, F.; Matsuura, M.; et al. The ALMA detection of CO rotational line emission in AGB stars in the Large Magellanic Cloud. AEA 2016, 596, A50. [CrossRef] 
46. Goldman, S.R.; van Loon, J.T.; Zijlstra, A.A.; Green, J.A.; Wood, P.R.; Nanni, A.; Imai, H.; Whitelock, P.A.; Matsuura, M.; Groenewegen, M.A.T; et al. The wind speeds, dust content, and mass-loss rates of evolved AGB and RSG stars at varying metallicity. MNRAS 2017, 465, 403-433. [CrossRef]

47. Bladh, S.; Höfner, S.; Aringer, B.; Eriksson, K. Exploring wind-driving dust species in cool luminous giants. III. Wind models for M-type AGB stars: Dynamic and photometric properties. AEA 2015, 575, A105. [CrossRef]

48. Bladh, S.; Liljegren, S.; Höfner, S.; Aringer, B.; Marigo, P. An extensive grid of DARWIN models for M-type AGB stars. I. Mass-loss rates and other properties of dust-driven winds. AEA 2019, 626, A100. [CrossRef]

49. Gros, M.; Crivellari, L.; Simonneau, E. An Implicit Integral Method to Solve Selected Radiative Transfer Problems. IV. The Case of Spherical Geometry. ApJ 1997, 489, 331-345. [CrossRef]

50. Crivellari, L.; Cristallo, S.; Piersanti, L. Universe Letters. In Preparation. Available online: https://journals.aps.org/prl/abstract/ 10.1103/PhysRevLett.108.225301 (accessed on 25 March 2011).

51. Ferrarotti, A.S.; Gail, H.P. Composition and quantities of dust produced by AGB-stars and returned to the interstellar medium. $A \mathcal{E} A$ 2006, 447, 553-576. [CrossRef] 